Research Paper

\title{
Clinical Significance of a Point Mutation in DNA Polymerase Beta (POLB) Gene in Gastric Cancer
}

\author{
Xiaohui Tan¹, 4 , Hongyi Wang ${ }^{2}$, Guangbin $\mathrm{Luo}^{3}$, Shuyang Ren¹, Wenmei Li1 ${ }^{1}$, Jiantao Cui ${ }^{1}$, Harindarpal S. \\ Gill4, Sidney W. Fu ${ }^{4}$, Youyong Lu ${ }^{1 凶}$ \\ 1. Laboratory of Molecular Oncology, Key Laboratory of Carcinogenesis and Translational Research (Ministry of Education); \\ 2. Department of Sugary, Peking University School of Oncology, Beijing Cancer Hospital \& Institute, Beijing, 100142, P.R. China. \\ 3. Department of Genetics, Case Western Reserve University, Cleveland, OH, 44106, USA. \\ 4. Department of Medicine, The George Washington University School of Medicine and Health Sciences, Washington, DC 20037, USA.
}

\Corresponding author: Sidney W. Fu (sfu@gwu.edu), or Youyong Lu (youyonglu66@sina.com)

(c) Ivyspring International Publisher. This is an open-access article distributed under the terms of the Creative Commons License (http://creativecommons.org/ licenses/by-nc-nd/3.0/). Reproduction is permitted for personal, noncommercial use, provided that the article is in whole, unmodified, and properly cited.

Received: 2014.10.01; Accepted: 2014.11.19; Published: 2015.01.01

\begin{abstract}
Gastric cancer (GC) is a major cause of global cancer mortality. Genetic variations in DNA repair genes can modulate DNA repair capability and, consequently, have been associated with risk of developing cancer. We have previously identified a $T$ to $C$ point mutation at nucleotide 889 (T889C) in DNA polymerase beta (POLB) gene, a key enzyme involved in base excision repair in primary GCs. The purpose of this study was to evaluate the mutation and expression of POLB in a larger cohort and to identify possible prognostic roles of the POLB alterations in GC. Primary GC specimens and their matched normal adjacent tissues were collected at the time of surgery. DNA, RNA and protein samples were isolated from GC specimens and cell lines. Mutations were detected by PCR-RFLP/DHPLC and sequencing analysis. POLB gene expression was examined by RT-PCR, tissue microarray, Western blotting and immunofluorescence assays. The function of the mutation was evaluated by chemosensitivity, MTT, Transwell matrigel invasion and host cell reactivation assays. The T889C mutation was detected in 18 (10.17\%) of $177 \mathrm{GC}$ patients. And the T889C mutation was associated with POLB overexpression, lymph nodes metastases and poor tumor differentiation. In addition, patients with- the mutation had significantly shorter survival time than those without-, following postoperative chemotherapy. Furthermore, cell lines with T889C mutation in POLB gene were more resistant to the treatment of 5 -fluorouracil, cisplatin and epirubicin than those with wild type POLB. Forced expression of POLB gene with T889C mutation resulted in enhanced cell proliferation, invasion and resistance to anticancer drugs, along with increased DNA repair capability. These results suggest that POLB gene with T889C mutation in surgically resected primary gastric tissues may be clinically useful for predicting responsiveness to chemotherapy in patients with GC. The POLB gene alteration may serve as a prognostic biomarker for GC.
\end{abstract}

Key words: DNA polymerase beta (POLB); gastric cancer; DNA Repair; point mutation; chemotherapy.

\section{Introduction}

Gastric cancer (GC) is the second leading cause of cancer-related deaths worldwide [1]. It remains difficult to cure, primarily due to lack of reliable screening markers and advanced disease when diagnosed. Even patients who present the most favorable condition at diagnosis and subsequently undergo curative surgical resection often die of recurrent dis- ease [2]. Genetic variations in DNA repair genes can modulate DNA repair capability and, consequently, have been associated with the risk of developing cancer [3]. DNA polymerase beta (POLB) is a DNA repair polymerase involved in base-excision repair (BER), and recombination and drug resistance [4-6]. Thirty percent of all tumors reported to date harbor 
mutations in the POLB gene [7]. Aberrant POLB expression resulted in an increased rate of spontaneous mutagenesis and a highly mutagenic tolerance phenotype $[8,9]$. POLB gene mutation and overexpression have been reported in various cancers [10-14]. In our previous studies, we identified a novel homozygous $\mathrm{T}$ to $\mathrm{C}$ point mutation at nucleotide 889 (T889C) in POLB gene (GenBank Acc\# M13140.1) from human primary GC. This mutation resulted in a substitution of the amino acid at 259 from Leucine to Serine in protein [15]. In the present work, we examined the T889C mutation and expression of POLB in a large cohort of GC patients with integral follow-up records, and analyzed the relationship between aberrant POLB expression and patients' clinicopathological features.

\section{Materials and Methods}

\section{Cell lines and tissue samples}

Eight human GC cell lines AGS, N-87, SUN1, SUN5, SNU16, RF1, RF48 and MKN45 were obtained from the American Type Culture Collection (ATCC). Cell lines BGC823, MGC803, SGC7901, PAMC82, and GES were established in China. Primary GC specimens and their matched normal adjacent tissues were collected and snap-frozen at the time of surgery. None of the patients had received any chemotherapy or radiotherapy before surgical resection. Clinical and histological features were obtained and evaluated (Table 1). Fifty peripheral blood samples were obtained from healthy volunteers. The study was conducted according to the Helsinki Declaration, approved by the Institutional Ethical Standards Committee of Peking University. Informed consent of all study subjects were obtained before the samples were collected.

\section{DNA isolation and PCR amplification}

Genomic DNA was extracted from GC cell lines, primary GC tissues and their matched normal gastric tissues, and peripheral blood samples of healthy volunteers. Since T889C mutation results in a new Taq1 site from 888 to 891 (TTGA to TCGA) in POLB gene, which allows us to screen for the point mutation by Taq1 enzyme digestion (Figure 1A). Specifically, we used a PCR strategy that can distinguish the T889C mutation from the wild-type in POLB allele. The primers used were: POLB Forward: 5'-CCACTTAGGCTTATCTTTGGT-3' and Reverse: 5'-GGA CAC TCA CCA GTG ACT CC-3'.

\section{Purification of PCR products and PCR-RFLP analysis}

The PCR products of the expected sizes were purified with Geneclean Kit (Bio 101, Inc., La Jolla, Calif.). Ten $\mu 1$ of PCR products were digested with the restriction enzyme, TaqI (New England BioLabs, Beverly, Mass.).

\section{PCR-DHPLC and DNA sequencing analysis}

DHPLC (denaturing high-performance liquid chromatography) analysis was performed as described [16, 17]. PCR products showing aberrant PCR-RFLP banding patterns and exhibiting abnormal elution peak in DHPLC were subjected to sequencing analysis.

\section{RT-PCR assays}

Five $\mu$ g of total RNA from each sample was used for cDNA synthesis, using the MMLV and random primers (Oligo dT). Two $\mu \mathrm{g}$ of reverse-transcription product was used as templates to amplify specific fragment of $P O L B$ gene. The expression of housekeeping gene glyceraldehydes-3-phosphate dehydrogenase $(G A P D H)$ was used as an internal control. The average relative densities of bands were detected by Bandlead 3.0 program. The expression level was shown by the ratio of POLB to GAPDH in band density.

Table 1. Clinical and pathological parameters of the 177 patient samples used in mutation analysis

\begin{tabular}{ll}
\hline Tissues parameters & Number \\
\hline Tumor & 177 \\
Gender & \\
Male & 125 \\
Female & 52 \\
Age (years) & \\
Male & $28-86$ \\
Female & $29-79$ \\
Pathology & \\
Intestinal & 116 \\
Diffuse & 30 \\
Mixed & 20 \\
Adenosquamous & 11 \\
Tumour location & \\
GOJ* & 51 \\
Fundus & 12 \\
Greater curve-body & 18 \\
Lesser curve-body & 11 \\
Body non-specified & 20 \\
Antrum & 65 \\
Differentiation & \\
Well & 21 \\
Moderate to poor & 60 \\
Poor & 96 \\
Depth of invasion & \\
Mucosa & 11 \\
Submucosa & 10 \\
Muscularis propria & 31 \\
Serosa/full thickness & 125 \\
No. of involved Lymph nodes & \\
0 & 60 \\
1 & 5 \\
$1-6$ & 19 \\
$7-15$ & 30 \\
$>15$ & 63 \\
\hline
\end{tabular}

* Gastro-esophageal junction 
Tissue microarray construction and immunohistochemical (IHC) staining and evaluation

Tissue microarrays were constructed as described previously [18]. An affinity purified goat polyclonal antibody POLB (sc-5927, Santa Cruz) was used for IHC staining. For evaluation, the total fields were counted microscopically under high power $(\times 200)$. The results of POLB expression were graded as: negative $(-),<5 \%$ of cell stained; positive $(+),>5 \%$ of cells stained.

\section{Western blot analysis}

Protein extraction and Western blot analysis with chemiluminescent detection were as described [19]. The following antibodies and dilution factors were used: affinity purified goat polyclonal antibody POLB (sc-5927, Santa Cruz, 1:800), rabbit anti-actin (H-196, Santa Cruz, 1:1000), donkey anti-goat IgG-HRP (sc-2020, Santa Cruz, 1:2000) and goat anti-rabbit IgG-HRP (sc-2030, Santa Cruz, 1:2000).

\section{Association analysis between alterations of POLB gene and patients' clinicopathological} features, and their responses to chemotherapy

The association between the point mutation and clinicopathological features such as gender, age, pathological diagnosis, differentiation, tumor location, depth of invasion, liver metastasis, and lymph metastases was analyzed by the paired chi-square test with SPSS 13.0. $p<0.05$ was considered statistically significant.

To analyze the correlation between T889C mutation and patients' response to postoperative adjuvant chemotherapy, we compared progression-free time and overall survival time of the patients who received identical anticancer drug treatment, at the same pathological stage when initially diagnosed.

Regarding surgical operations, patients included for this study must have received complete surgical removal of the tumor, with negative pathologic margin, and with the caveat of the anatomical location of the tumor and the condition of the patients. The degree of surgical resection was assessed by a central review of operative reports, and pre- and post-operative imaging. When the results of scans and operative reports differed, the degree of surgical resection was assessed based on the results of computed tomography (CT) or magnetic resonance imaging (MRI). Patients with one of the following two conditions were excluded: 1 . underwent unsuccessful surgical procedures (for instance, anastomotic leakage, fistula, abscess, hemorrhage); 2 . had "medical" complications caused by non-malignant diseases or uncontrolled infections, which were detected by the following examinations: CT, MRI, height, weight, com- plete blood count, hemoglobin level, kidney/liver function, electrolyte levels, and calcium and magnesium concentrations, and a caloric intake per day by oral route $(<1500 \mathrm{kcal}$ were excluded).

Regarding postoperative adjuvant chemotherapy, patients eligible for the analysis should have a complete and detailed medical record and a report with gastroscopy, pathological, CT or MRI diagnosis, quality-of-life assessment, progression-free survival and overall survival time. And the information was obtained before chemotherapy and at specific times during and after drug treatment. Cases that were unclear whether abnormalities on postoperative scans represented residual tumor or postoperative artifacts, and cases with combination therapy of chemotherapy, immunotherapy and/or radiotherapy were all excluded.

Judgments of disease relapse or progression were based on clinical evidence of gastroscopy, pathological, CT or MRI diagnosis. Patients who had clinical evidence of disease progression, despite scans indicating otherwise, were considered disease progression. The site and date of first relapse, and the cause and date of death were recorded. The sites of relapse were classified as follows: the relapse was coded as: loco-regional if tumor was detected within the operation fields (including gastric bed, remnant stomach, and surgical anastomosis); peritoneal if tumor was detected in the peritoneal cavity; and distant if the metastases were diagnosed outside the peritoneal cavity or liver metastasis.

\section{Real time qRT-PCR analysis}

First-strand cDNA was synthesized using the Bio-Rad RT kit. Primer sequences are available upon request. Real-time qRT-PCR assays were carried out on a Bio-Rad iCycler iQ system (Bio-Rad, Hercules, CA, USA) using SYBR Green reagent as described [19].

\section{Construction of eukaryotic expression vector containing wild type and T889C mutants of POLB gene and transient transfection}

The entire coding region of POLB cDNA containing wild type or T889C mutant, and site-directed mutagenesis were amplified by RT-PCR, and cloned respectively in a pcDNA3.1 vector before transfecting into AGS cells. $5 \times 10^{5}$ cells were plated in $10 \mathrm{~cm}^{2}$ culture dishes, 25 pg of plasmid DNA/dish were transfected using the calcium phosphate precipitation method.

\section{Immunofluorescence assays}

Cells were seeded at $2 \times 10^{4}$ cells per well on glass coverslips in six-well plates and fixed in $2 \%$ 
paraformaldehyde as described previously [19]. Confocal images were obtained using a LSM 510 Confocal microscope (Carl Zeiss). The number of nuclei containing at least one localized area of immunofluorescence was determined by examination of the confocal images. Antibodies for immunofluorescence were as follows: goat polyclonal antibody $P O L B$ (sc-5927, Santa Cruz) at final concentration of 1:800, Alexa Flour 568 goat anti-mouse IgG (Invitrogen), 1:500 and Alexa Flour 568 goat anti-mouse IgG (Invitrogen), 1:500.

\section{POLB knockdown assays}

The siRNA targeting POLB was purchased from Qiagen (SI02663605: 5'-TACGAGTTCATCCATCA ATTT-3'). siRNAs against GAPDH (SI02653266 and SI03650325, Qiagen) were used as positive and negative controls, respectively. Cells were transfected with 40 pmol of siRNA using the HiPerFect lipid transfection reagent (Qiagen), and harvested at $24 \mathrm{~h}, 48 \mathrm{~h}$ and $72 \mathrm{~h}$ post-transfection. POLB expression was then analyzed at both mRNA and protein level by qRT-PCR and Western blotting, respectively.

\section{Chemosensitivity and MTT assays}

To determine the sensitivity of GC cell lines to chemotherapy, BGC823, SGC7901, AGS and N87 cells were seeded in a 96-well plate as described [20]. 5-FU, cisplatin and epirubicin were obtained from the pharmacy at Beijing Cancer Hospital (Beijing, China). Test drug concentrations were 5 to $400 \mu \mathrm{g} / \mathrm{mL}$ for 5-FU, 0.5 to $8 \mu \mathrm{g} / \mathrm{mL}$ for cisplatin and 0.025 to 1.6 $\mu \mathrm{g} / \mathrm{mL}$ for epirubicin. The cells were treated by either single or a variety of combinations for analysis. Cells were seeded in a 96-well plate at the density of $3 \times 10^{4}$ per well, and were incubated in medium containing $5 \%$ serum. The attached cells in the plates were washed once with PBS, and then replaced with fresh medium containing various concentrations of drugs to treat for $24 \mathrm{~h}$ and $48 \mathrm{~h}$. To further confirm the effect of the T889C on chemotherapy, the wild type, T889C mutant or empty vector transfected AGS cells were subject to MTT (3-(4,5-dimethylthiazol-2-yl)-2,5diphenyltetrazolium bromide) assays at $24 \mathrm{~h}, 48 \mathrm{~h}$ and 72h. At each time point, $10 \mu \mathrm{l}$ of MTT reagent (MILLIPORE, Billerica, MA, USA) was added to each well. After $4 \mathrm{~h}$ of incubation, cells were lysed by addition of $200 \mu \mathrm{l}$ DMSO. Absorbance was measured at $570 \mathrm{~nm}$ wavelength. Experiments were repeated three times, and the percentage of growth was the average of the experiments.

\section{Transwell matrigel invasion assays}

The cell line AGS transfected with wild-type and mutant $P O L B$ was harvested using a cell-dissociation solution at $90 \%$ confluence. The cells $\left(1 \times 10^{5}\right)$ were suspended in $100 \mu \mathrm{l}$ of serum-free medium before adding to the upper chamber of the Transwell insert. The lower chamber was filled with $500 \mu \mathrm{l}$ of the medium with $0.1 \%$ bovine serum albumin. After $24 \mathrm{~h}$ of incubation at $37^{\circ} \mathrm{C}$, the cells on the upper surface of the filter were removed using a cotton swab. The cells that penetrated to the lower surface of the filter were stained with hematoxylin and counted under an Olympus IX70 microscope (Olympus) in 13 randomized fields at $\times 400$ magnification. The assay was performed four times as described earlier [20,21].

\section{Plasmid treatment with UV/anticancer drugs and host cell reactivation (HCR) assays}

DNA repair capability of cells with wild-type and T889C mutant POLB were assessed using post-UV HCR as described previously [19] [20]. pCMVLuc reporter gene plasmid (a kind gift from Dr. Kenneth H. Kraemer, National Cancer Institute ) was dissolved in $10 \mathrm{~mm}$ Tris-HCl, $1 \mathrm{~mm}$ EDTA, $\mathrm{pH} 8$ (TE buffer) to a final concentration of $100 \mu \mathrm{g} / \mathrm{ml}$ and poured in a Petri dish to form 1D $2 \mathrm{~mm}$ thick layer. The Petri dish was placed on ice and irradiated by $1000 \mathrm{~J} / \mathrm{m}^{2}$ of UV light. For the drug treatment, $1 \mu \mathrm{l}$ aliquots of a stock solution of $1 \mu \mathrm{g} / \mu \mathrm{l}$ cisplatin, $10 \mu \mathrm{g}$ / $\mu \mathrm{l} 5$-FU and $0.01 \mu \mathrm{g} / \mu \mathrm{l}$ cisplatin (Sigma-Aldrich, St. Louis, MO) in TE were added to $10 \mu \mathrm{g}$ plasmid DNA before dissolving in $200 \mu \mathrm{l}$ TE buffer, and then the samples were incubated at $37^{\circ} \mathrm{C}$ for $6 \mathrm{~h}$. At the end of the incubation, $1 \mathrm{M} \mathrm{NaCl}$ was added to a final concentration of $0.2 \mathrm{M} \mathrm{NaCl}$. The plasmid DNA was precipitated with 2 volumes of $100 \%$ ethanol, and then washed with $70 \%$ ethanol. The pellets were air-dried and dissolved in TE buffer. DNA repair capability of cells was assessed using HCR with the CsCl-purified pCMVLuc plasmid treated by UV or drugs. Four microliters (200 ng) of damaged or undamaged pCMVLuc were co-transfected with $0.4 \mu \mathrm{l}(200 \mathrm{ng})$ of wild-type, and T889C mutant POLB effector plasmids into cells using Lipofectamine 2000 (Invitrogen). To estimate the DNA repair capacity after POLB knockdown, we co-transfected pCMVLuc with 40 pmol of siRNA, relative luciferase activities were determined by a percentage of activities obtained with treated versus untreated control plasmids.

\section{Statistical analysis}

Means \pm SD were calculated, and statistical analysis was performed using one-way ANOVA, followed by the nonparametric Kruskal-Wallis test; $p<$ 0.05 was considered statistically significant.

\section{Protein-structure modelling}

A three-dimensional model of the L259S mutation was created by comparative protein modelling methods [22]. The crystal structure of human POLB 
(PDBid: 4JWM) at 2.0- $\AA$ resolution [23] was used as a template as justified by a $98.5 \%$ identity in amino-acid sequence alignment. Using the model-building program O [24, 25], residue E256 was replaced with aspartate to restore the active site back to that of the wild type, and residue L259 was replaced with serine to model the natural occurring mutation in cancer patients. The peptide backbones were not adjusted. Side chains were adjusted based on likelihood of nearby hydrogen-bonds partners within a distance of $\sim 3.5$ A. Protein residue numbering corresponds to the coding sequence within genbank accession M13140.1.

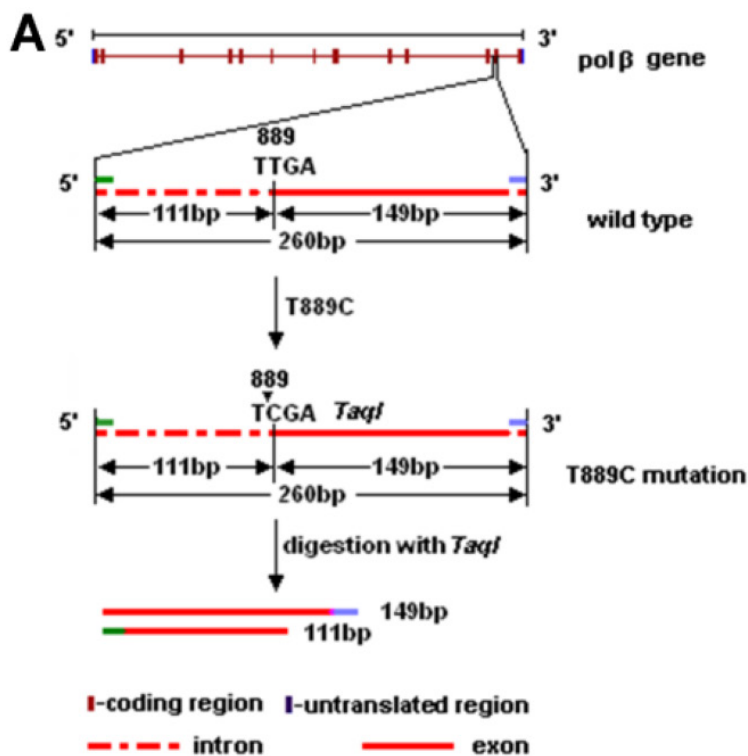

B

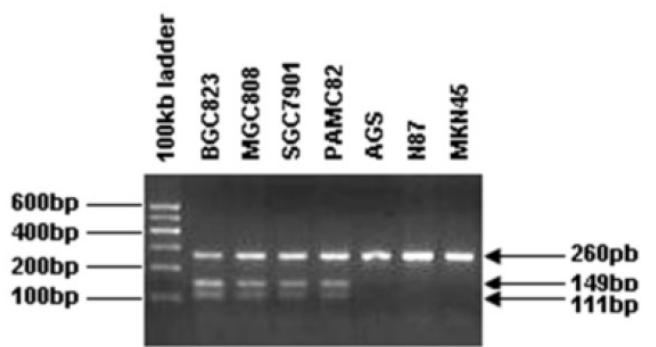

\section{Results}

\section{T889C point mutation screening in a new co- hort of GC patients}

In order to investigate the incidence of $\mathrm{T} 889 \mathrm{C}$ mutation in GC, we established a new cohort of 177 GC patients (Table 1). To facilitate large-scale T889C point mutation screening, a strategy based on PCR-RFLP was designed to characterize the mutation (Figure 1A). The feasibility of this strategy was firstly tested in 13 GC cell lines. PCR products from cell lines with T889C mutant POLB, BGC823, MGC803, SGC7901 and PAMC82 [15] were able to be cut by Taq1, but not for those with wild type POLB, AGS, N87 and MKN45. Thus, the PCR-RFLP indeed provided a reliable and simple approach for detecting the T889C mutation (Figure 1B).
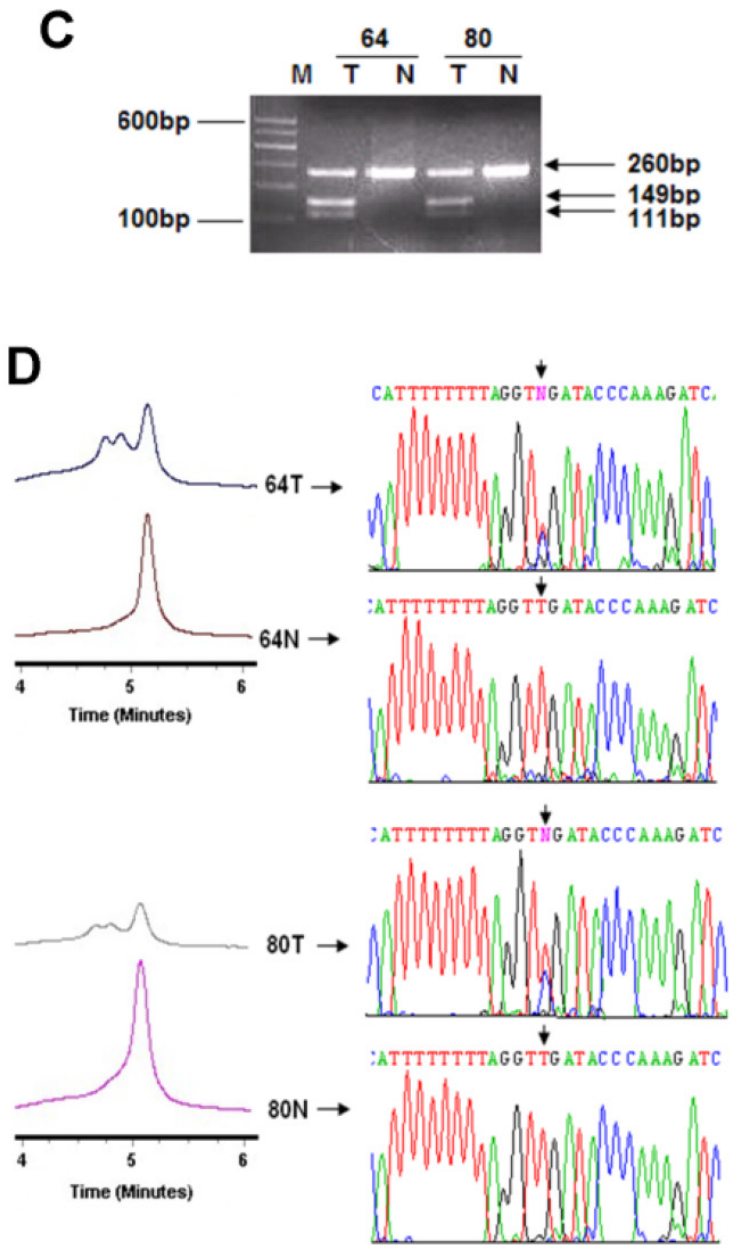

Figure 1. Identification of the T889C mutation in primary GCs with PCR-RFLP, DHPLC and DNA sequencing. A. Restriction analysis of the POLB gene fragment amplified by PCR. RFLP patterns obtained after digestion of PCR products with Taql endonucleases. Primers were designed to amplify the region spanning 3 ' region of intron 12 to 5 ' region of exon 13 in POLB gene and length of the PCR products were $260 \mathrm{bp}$. T889C mutation formed of a new Taql site from nucleotide 888 to 891 (TTGA to TCGA) of POLB (NM 002690.1) cDNA and therefore, the amplified product could be digested with Taql. The molecular weights of digested fragments were 149bp and $111 \mathrm{bp}$. B. The digested fragments were separated by $2.0 \%$ agarose gel electrophoresis. 100-bp ladder was used as a size marker. PCR products (260bp) amplified from BGC823, MGC803, SGC7901 and PAMC82 cell lines (carrying the T889C mutation) could be digested by Taq I, the molecular weights of digested fragments were 149 and 111 bP while those from AGS, N87 and MKN45 cell lines couldn't be digested by Taq I. C. Restriction patterns obtained by Taq I digestion of amplified product from GC tissues. The digested fragments were separated by $2.0 \%$ agarose gel electrophoresis and $100 \mathrm{bp}$ ladder was used as a size marker. PCR products (260bp) with T889C mutation could be digested by Taql, the molecular weights of digested fragments were 149 and $111 \mathrm{bp}$ while the PCR products with wild type POLB couldn't be digested by Taq I. D. Chromatograph of DHPLC and sequencing analysis. 
We examined 177 primary GCs. The T889C point mutation was detected in $18(10.17 \%)$ of them (Figure $1 C)$, but not in any of the matched normal gastric tissues or the 50 normal volunteer controls (Figure 1D). These data indicate that $\mathrm{T} 889 \mathrm{C}$ mutation may represent an important genetic risk factor for human GCs.

Since point mutations could alter gene function by either reducing (loss-of-function mutations) or increasing (gain-of-function mutations) its expression level, we examined the relationship between T889C mutation and expression of POLB in GC tissues. We constructed tissue microarrays consisting of samples from 70 GC patients, including 18 with- and 52 without- T889C mutation, and 20 normal adjacent tissues (5 from patients with- and 15 without- T889C mutation). The positive hybridization signals of $P O L B$ protein were observed in $47(67.1 \%)$ of the $70 \mathrm{GCs}$, of which, $16(88.9 \%)$ of 18 with T889C mutation, and 31 (59.6\%) of 52 without. There was a significant positive association between T889C point mutation and positive signals of POLB protein $(p=0.023$, Figure 2A-F and Supplementary Material: Table S1). The concordance between T889C mutation and overexpression of POLB was confirmed by RT-PCR and Western blot analysis, respectively (Figure $2 \mathrm{G} \& 4 \mathrm{C}$ ).

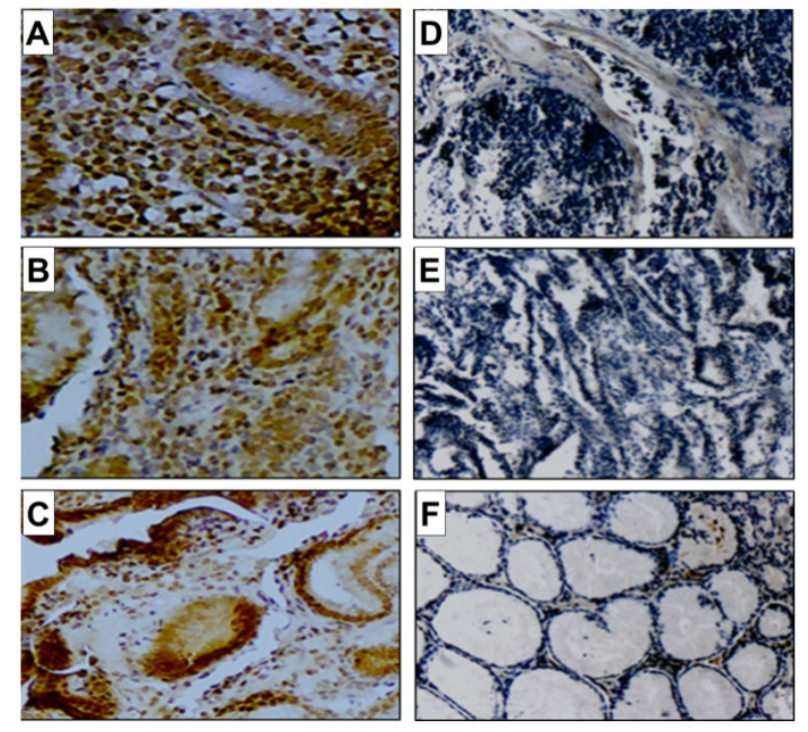

G

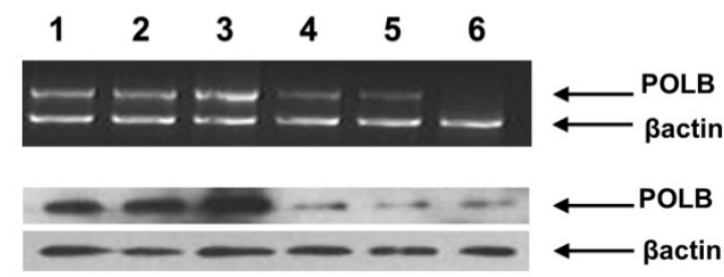

Figure 2. POLB expression on tissue array by immunohistochemistry, RT-PCR and Western blot analysis in GC patients. A, B and C. With T889C mutation; D and E. Without T889C mutation; F. Normal tissue. G. RT-PCR (top) and Western blot (bottom) analysis of POLB gene in GC tissues with-T889C mutation (lanes 1-3) and without- (lanes 5-6).

\section{Association of clinicopathological features with T889C mutation and POLB overexpres- sion}

We analyzed the relationship between T889C mutation and gender, age, tumour location, depth of invasion, differentiation and metastasis in 177 primary GCs. Lymph node metastases was found in all 18 GC patients carrying T889C mutation (100\%), whereas in 99 of the rest of the 159 patients $(62.26 \%)$. There were significant differences between the patients with- and without- $\mathrm{T} 889 \mathrm{C}$ mutation in regard to the propensity of lymph nodes metastasis $(p=0.002$, Supplementary Material: Table S2). GC harboring T889C mutation was more likely to metastasize to lymph nodes. Furthermore, mutation occurred in 14 $(14.58 \%)$ of 96 poorly differentiated primary GCs and $4(4.94 \%)$ of 81 moderately/well differentiated tumors $(p=0.034$, Supplementary Material: Table S2). Thus the T889C mutation is more prevalent in poorly differentiated primary GCs compared with those of moderately/well differentiated tumors. However, we did not find any significant correlation between T889C mutation and gender, age, tumour location or depth of invasion $(p>0.05)$. These findings suggest that $\mathrm{T} 889 \mathrm{C}$ mutation is strongly associated with metastasis and differentiation in human GC.

We next analyzed the relationship between $P O L B$ expression and clinicopathological features. By analyzing the 70 samples on our tissue array, we found that $P O L B$ positivity was not associated with lymph nodes metastasis, poorly differentiation, age, gender, tumour location and depth of invasion $(p>$ $0.05)$, despite that T889C mutation was significantly associated with poor differentiation $(p=0.009)$ and lymph nodes metastasis $(p=0.018)$. Our data suggested that $P O L B$ expression alone is not associated with clinicopathological features.

\section{T889C mutation is associated with the re- sponse to postoperative chemotherapy}

POLB plays a significant role in chemotherapeutic agent resistance [26]. Among the 177 cases, 143 patients received postoperative adjuvant chemotherapy. Nineteen GC patients were analyzed to reveal the relationship between the mutation and the response to postoperative chemotherapy as they were diagnosed at the same clinicopathological stage) before surgery, and treated with the same anticancer drugs/doses. They were all at stage T4N2M0, and treated with cisplatin $\left(60 \mathrm{mg} / \mathrm{m}^{2}\right.$ i.v. $)$ and epirubicin (50 mg / $\mathrm{m}^{2}$ i.v.), every 3 weeks for four cycles together with a continuous 12 week infusion of 5-FU (200 mg / $\mathrm{m}^{2}$ / day). The estimated median progression-free and overall survival times were $11 \pm 1.405$ and $16 \pm 4.265$ months, respectively. Significant differences 
in progression-free and survival time were found between patients with T889C mutation (8) and those without the mutation (11) $(p=0.026)$, and significant difference also existed in overall survival $(16 \pm 4.265$ vs. $24 \pm 8.34, p=0.027$ ) between the two groups (Figure $3 \mathrm{~A} \& \mathrm{~B})$. Patients with T889C mutation had significantly shorter survival than those without the mutation following chemotherapy. Our data indicates that T889C mutation is associated with poor response to postoperative chemotherapy.

To further examine the relationship between alternative $P O L B$ and poor response to anticancer drugs, we assessed the sensitivity of cell lines BGC823, SGC7901, AGS and N87 to the single-agent and combinations of 5-FU, cisplatin and epirubicin by MTT assays. We found that the cell lines with T889C mutation (BGC823, SGC7901) were more resistant to 5-FU, cisplatin and epirubicin than those with wild type POLB (Figure $4 \mathrm{~A} \& \mathrm{~B}$ ). These results support the clinical observation that T889C mutation is associated with poor response to postoperative chemotherapy.

A

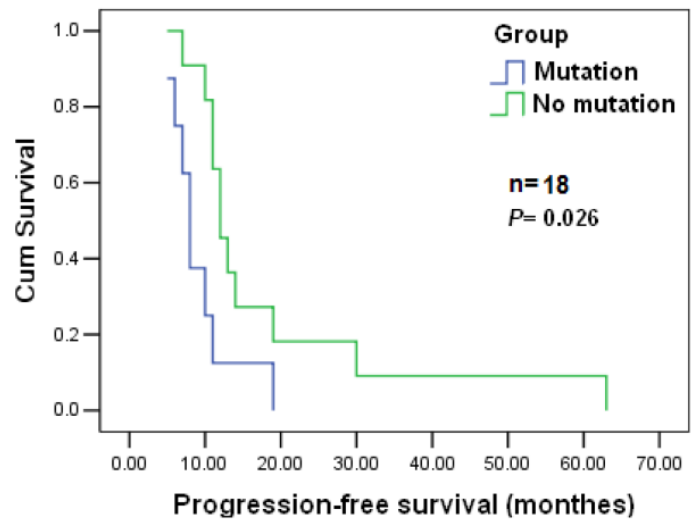

B

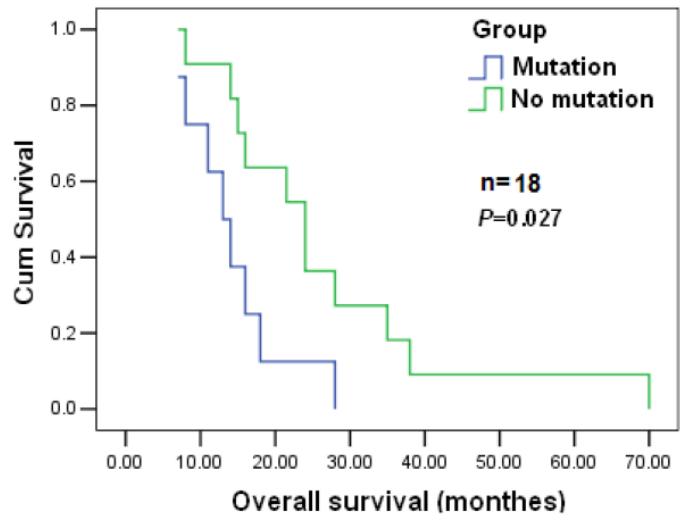

Figure 3. Correlation between T889C mutation and survival. Progression-free survival (top) and overall survival (bottom) for all 19 patients enrolled. The statistical significance of the difference was $p=0.027$ and $p=0.026$. Patients with T889C mutation had significantly shorter survival than did patients without the mutation after postoperative chemotherapy.
Overexpression of POLB gene stimulates proliferation and promotes invasion and migration in human GC cell lines

Having demonstrated the relationship between T889C mutation, and lymph node metastasis and differentiation in clinical samples, we further examined the role of $\mathrm{T} 889 \mathrm{C}$ mutation in metastasis, differentiation and resistance to anticancer drugs in GC cell lines. Relative POLB mRNA and protein levels in transfected AGS cell lines with wild type or T889C were significantly increased $(p<0.01)$ than that of the vector control lines, and the receptor genes expressing both the wild-type and mutant were localized to the nucleus (Figure 5A). We compared the number of cells expressing wild type or mutated POLB at 24, 48 and $72 \mathrm{~h}$ after transfection. The number of mutant $P O L B$-transfected cells increased more significantly than that of the wild-type (Figure 5B). Conversely, knockdown of POLB by siRNA resulted in a decreased cell growth in AGS cells. Our data suggested that cells transfected with T889C mutated POLB increased cell proliferation. Next, we examined the invasive and spontaneous metastatic potential by matrigel assays. The invasion of the mutant $P O L B$-transfected cells was significantly increased compared with the wild type POLB-transfected cells. In addition, knockdown of POLB by siRNA reduced cell growth in AGS cells (Figure 5C). These results suggest that GC cells with T889C mutation are more proliferative and invasive.

\section{Expression of POLB with T889C mutation leads to resistance to chemotherapeutic agents}

In order to further elucidate relationship between T889C mutation and resistance to chemotherapy, we transfected the AGS cell line with either wild type or T889C mutated POLB, and treated them with various concentrations of anticancer drugs. We found that cells transfected with T889C mutant were significantly more resistant to cisplatin and 5-FU compared to those transfected with empty vectors (Figure 5D). Inhibition of $P O L B$ expression by siRNA restored chemosensitivity in AGS cells. Our data further corroborates that T889C mutation correlated with poor response to postoperative chemotherapy.

\section{Overexpression of POLB with T889C mutation significantly increased post-drugs host cell re- activation activity in $\mathbf{G C}$ cell line}

POLB participates in regulation of DNA repair during carcinogen exposure [8]. We then asked whether overexpression of POLB increases the resistance of the GC cell lines to anticancer drugs via DNA repair pathway. We measured the luciferase 
activity by co-transfecting wild type or T889C mutant $P O L B$, along with pCMU-Luc vector [19], which was pre-treated by anticancer drugs respectively, into the AGS cell line. We found that AGS exhibited significantly increased luciferase activity when the pCMU-Luc vector was treated with cisplatin and 5-FU. There was no difference in luciferase activity when the pCMU-Luc vector was treated with epirubicin (Figure 5E). Our data indicate that the T889C mutation enhances the DNA repair capability in GC cell line.

When co-transfected AGS with POLB siRNA and pCMU-Luc vector pre-treated with anticancer drugs,

A

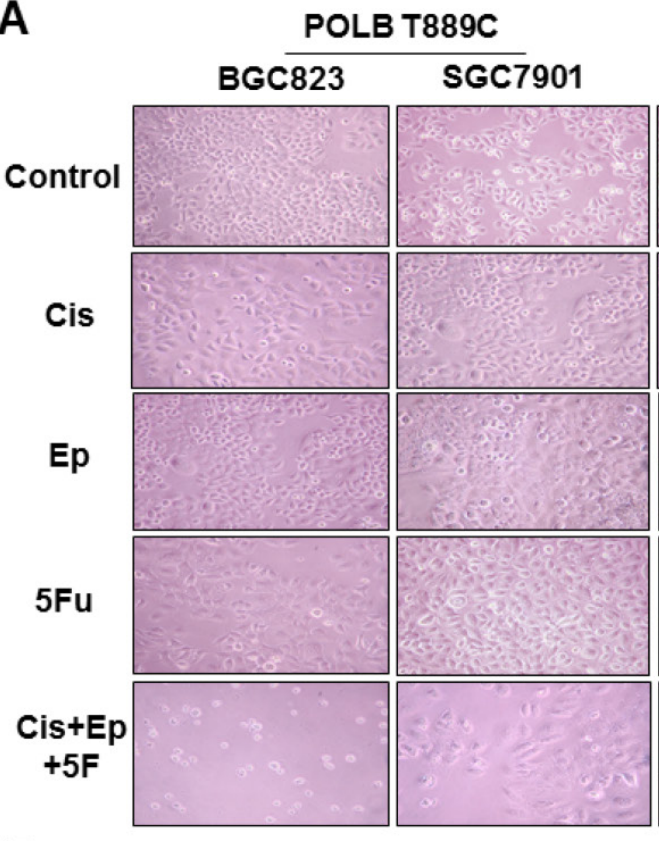

B
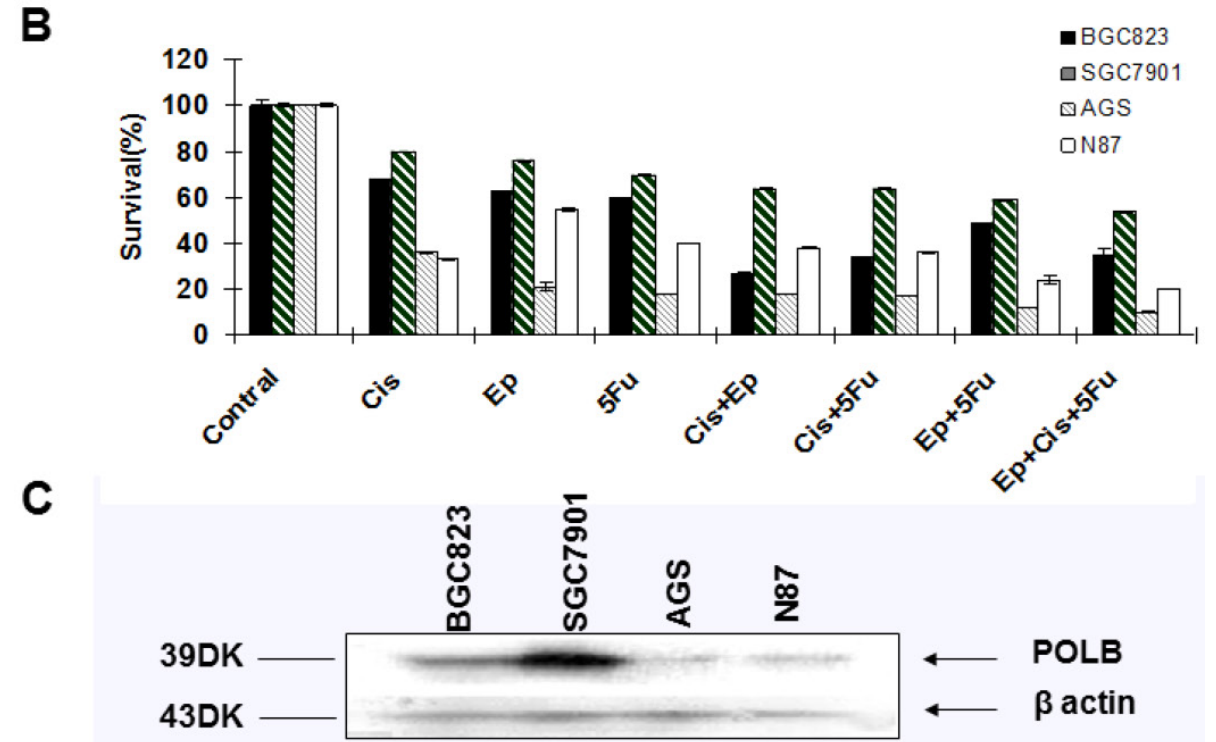

Figure 4. Correlation between T889C mutation and cytotoxicity of 5-fluorouracil, cisplatin and epirubicin. A. Phenotypic comparison of BGC823, SGC7901 and AGS cell lines before and after treatment. B. Sensitivity of cell lines to cisplatin, epirubicin, 5-fluorouracil and combination treatment with three drugs. Concentration of 5 -fluorouracil, cisplatin, epirubicin, and was $5 \mathrm{ug} / \mathrm{ml}, 0.5 \mathrm{ug} / \mathrm{ml}$ and $0.1 \mathrm{ug} / \mathrm{ml}$ respectively. The concentration of combination treatment of three drugs is cisplatin $0.5 \mathrm{ug} / \mathrm{ml}$, epirubicin $0.1 \mathrm{ug} / \mathrm{ml}$, and 5 -fluorouracil $5 \mathrm{ug} / \mathrm{ml}$. Survival is expressed as the relative plating efficiency of treated cells to the control. The values shown in the figures are mean readings from six wells in each experiment and representative of at least three independent experiments. C. Protein expression of POLB in cell lines BGC823, SGC7901, AGS and N87. 


\section{A hypothetical model of the human POLB with T889C mutation}

To elucidate the possible mechanism of the POLB T889C mutation associated functional changes, we modeled its protein crystallographic structure. The T889C mutation in the nucleotide sequence of genbank accession M13140.1 would result in an encoded serine at residue position 259 that would be in hydrogen-bonding proximity to carboxyl groups of residues 232 and 233 on the backbone chain in an adjacent $\beta$-strand (Figure 6). These interactions would distort the $\beta$-strand where the serine resides resulting in a positional shift of the catalytic residue, D256. In wild type, D256 is stabilized by one of the two bound metal ions $(\mathrm{Mg} 2+)$ and is thought to deprotonate the O3' group of the primer or newly forming DNA strand during synthesis [23]. A change in the active site could either result in a lower activation barrier for catalysis or simply loosen the constraints in the active site for the DNA strand to pass with greater flexibility and speed. There may be additional precedence for a more efficient active site as other hydrophobic substituted residues, now capable of electrostatic interactions, are also present around the active site, such as L228R, I260Y, and possibly L287K when comparing genbank accession number M13140.1 with AAA60133.1 used in our model (PDBid:4JWM).

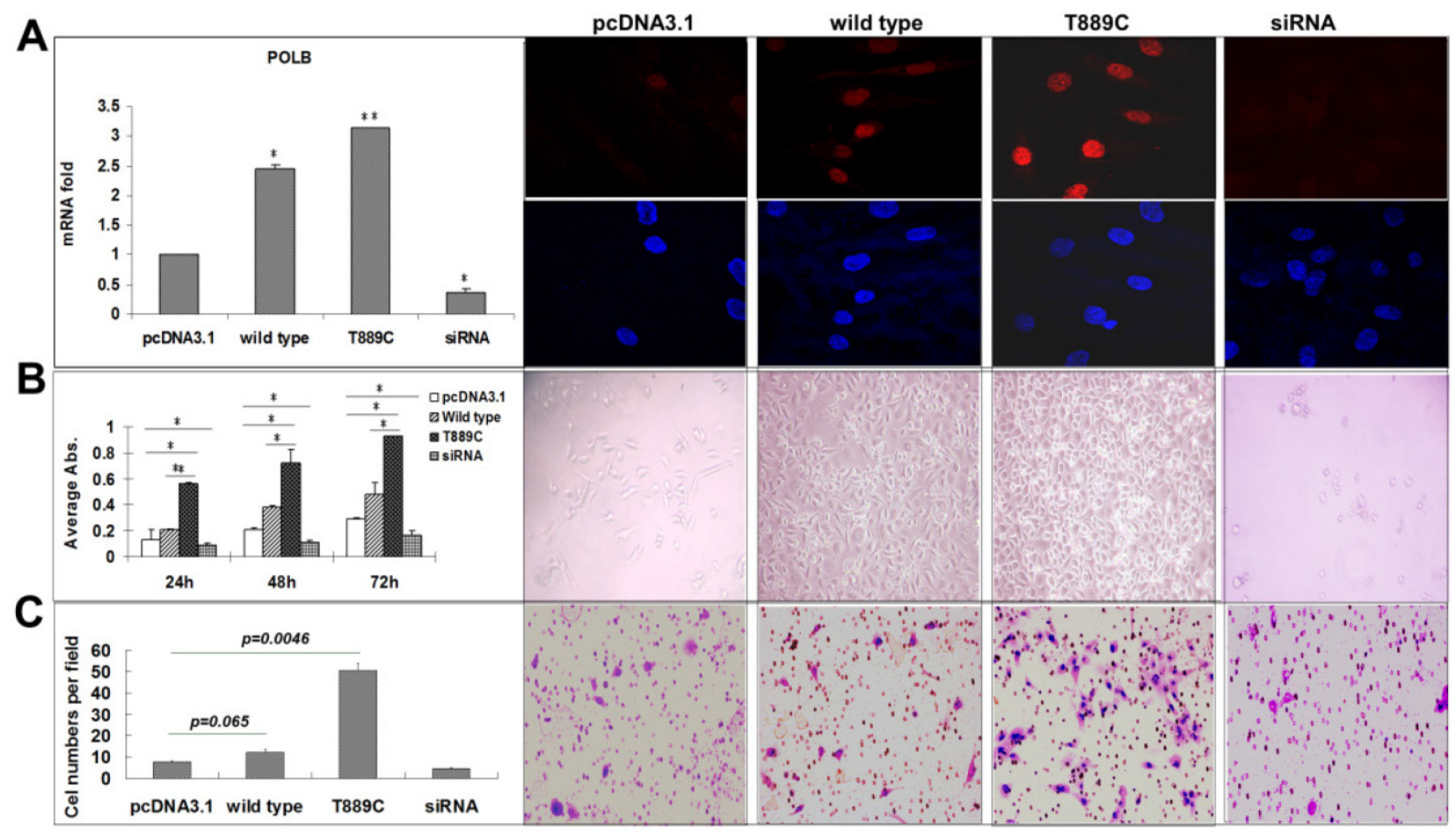

D

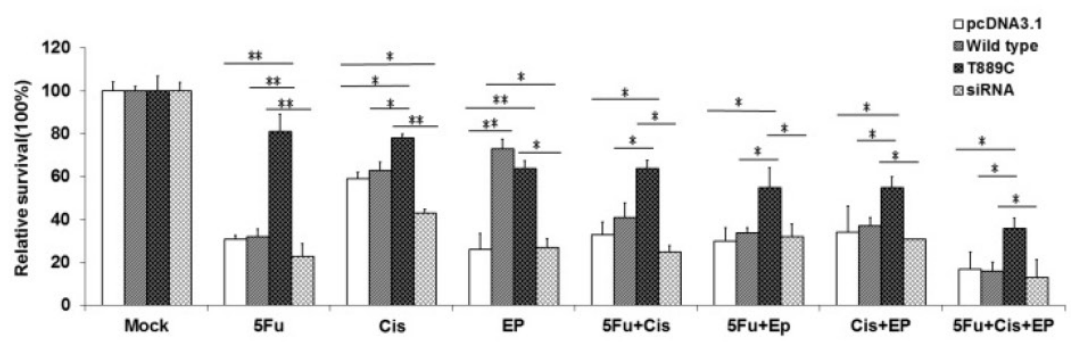

E

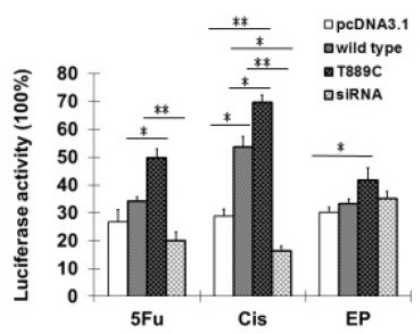

Figure 5. The effect of POLB overexpression. AGS cells were transfected with empty pCDNA3.1, wild type POLB, T889C mutant and POLB siRNA. A. Expression of transfected POLB genes in human GC cell lines. Left panel, representative results of the QRT-PCR analysis of POLB mRNA levels in AGS cell lines. Each reaction was run twice in triplicates. POLB expression was normalized by GAPDH. Right panel, representative results of the Immunofluorescence for POLB protein expression. POLB protein of both the wild-type and mutant localized to the nucleus. B. The effect of POLB wild type, mutant and siRNA in AGS cell proliferation. MTT assays indicate cell proliferation rate was significantly higher in T889C overexpressors (left). The experiments were done three times in triplicates. Right panel shows phenotypic comparison of AGS cell lines before and after transfection of POLB wild type and mutant and siRNA. C. Matrigel analysis. Three fields of unit area on each membrane were counted for cell numbers, and the experiments were repeated twice. Data analysis was based on the average of parallel repeats. T889C mutation overexpressing cell lines exhibited very significantly higher invasiveness than wild type and empty vector control cell lines. D. Resistance assay. The cells were treated with or without 5 -fluorouracil, cisplatin, and epirubicin for $24 \mathrm{~h}$. The values are represented as a mean from three separate experiments; bars, \pm SD. E. HCR assay showing increased post-UV DNA repair capability in T889C overexpressors. * $p<0.05$. 
A.

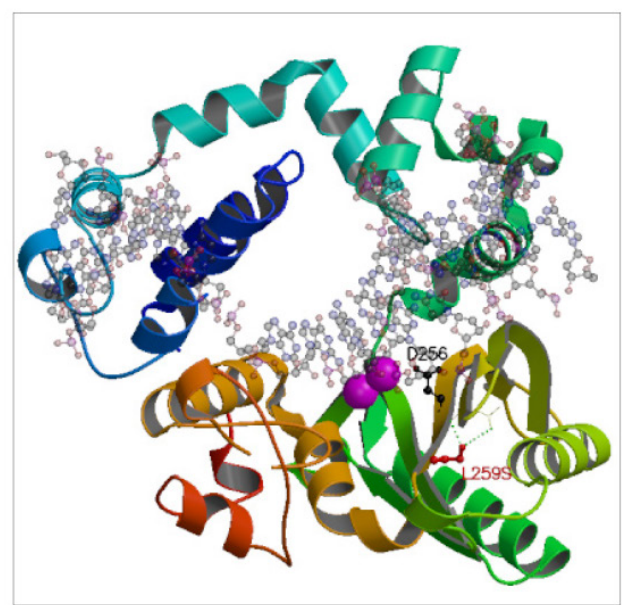

B.

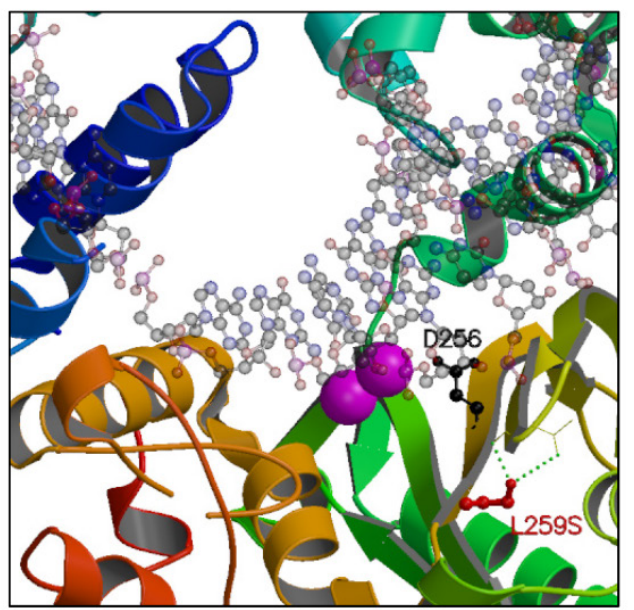

Figure 6. A hypothetical model of the human POLB enzyme is shown in complex with DNA, and containing the L259S mutation that is found commonly in gastric cancer patients. The two known metal ions bound in the active site are shown as magenta spheres. A. The entire enzyme is shown revealing the relative location of the serine in proximity to the active site. B. A close-up view of additional hydrogen-bond networks is shown in a $\beta$-sheet that would lead to a predicted small distortion in the active site. The figure was made with the program, MOLSCRIPT [38].

\section{Discussion}

This study presents a comprehensive picture of the T889C mutation in $P O L B$ gene and its expression in relation with GC patients' clinicopathological features. We deciphered the functional relationship between mutant and wild type POLB gene, in association with DNA repair pathway. The T889C mutation was associated with $P O L B$ overexpression, clinicopathological features, such as lymph node metastasis and differentiation, and response to chemotherapy. Our clinical and experimental data indicate an oncogenic property in mutant $P O L B$ gene in comparison to the wild type $P O L B$ gene in GC. Previous study suggested that $P O L B$ exhibits dichotomous functions depending on its expression, either as an oncogene or as a tumor suppressor [27-30]. Our data indicate that T889C mutation in POLB is a relatively frequent phenomenon and this may be an underlying mechanism for gastric cell mutagenesis via enhanced DNA repair pathway. There are two possible reasons where the L259S substitution may arise naturally. First, it could allow for higher expression levels. We found a significant positive association between T889C mutation and overexpression of $P O L B$ gene. However, the exact mechanism by which T889C mutation leads to POLB gene overexpression is not clear. We hypothesize that epigenetic factors may be involved, which would be our direction for future investigation. Second, beyond epigenetics, a serine substitution could conceivably increase the specific activity of the polymerase in crisis. Detailed X-ray crystallographic studies are needed to determine the effects of the substitutions in the active site.
DNA repair and the fidelity of DNA relegation have been found to be correlated with metastatic potential [31-33]. We discovered that T889C mutation, but not POLB overexpression alone, was significantly correlated to increased lymph nodes metastasis. Our data suggests that abnormally enhanced DNA repair capability increases the risk of tumor metastasis for GC.

Previous studies suggested that $P O L B$ may play a fundamental role in cellular differentiation [34, 35]. Our results further demonstrated that poorly-differentiated tumors carried higher frequency of T889C mutation than moderately/well differentiated tumors. We hypothesize that the poor differentiation associated with T889C mutation may be due to the disruption of DNA repair synthesis or DNA translation synthesis during differentiation, or by facilitating accumulation of mutations in differentiation-related genes.

POLB has been found to efficiently bypass the cisplatin adduct [36]. Studies showed that abnormal POLB expression associated with the cytotoxic effects of DNA damaging chemotherapeutic agents [37]. In this study, we showed that T889C mutation of POLB gene was correlated closely with a poor response to 5-FU, cisplatin and epirubicin in GC patients. It is suggested that T8889C mutation might facilitate cell survival by enhancing DNA repair capability, to bypass various DNA damages by commonly used chemotherapeutic drugs.

\section{Conclusions}

The T889C mutation in POLB gene is a prevalent event among GC patients. The alteration of POLB 
gene mutation and expression is associated with the pathological features including differentiation, lymph nodes metastasis, and poor prognosis via DNA repair pathway. Thus, the POLB gene with T889C mutation in surgically resected primary gastric tissues may be clinically useful for predicting responsiveness to chemotherapy in patients with GC. The POLB gene alteration may serve as a prognostic biomarker for GC.

\section{Abbreviations}

GC, Gastric cancer; POLB, DNA polymerase beta; PCR-RFLP, PCR-restriction fragment length polymorphism.

\section{Supplementary Material}

Tables S1 -S2. http://www.ijbs.com/v11p0144s1.pdf

\section{Acknowledgements}

We would like to thank Dr. Kenneth H. Kraemer (NCI/NIH) for providing us with the pCMVLuc reporter gene plasmid and thank the individual patients for participating in this study. This work was supported by grants from the National Key Basic Research Program Project of China (Grant\# 2004CB518708), the National Natural Science Foundation of China (NSFC) (Grant\# 39625016), the National Bio-Tech 863 Program (Grant\# 2012-AA02A203), the NIDDK/NIH (K01DK082646 to HSG) and the Elaine H. Snyder Cancer Research Award (to SWF).

\section{Competing Interests}

The authors have declared that no competing interest exists.

\section{References}

1. Shi Y, Li L, Hu Z, Li S, Wang S, Liu J, et al. A genome-wide association study identifies two new cervical cancer susceptibility loci at 4q12 and 17q12. Nat Genet. 2011; 45: 918-22. doi:10.1038/ng.2687.

2. Liu X, Cai H, Shi Y, Wang Y. Prognostic factors in patients with node-negative gastric cancer: a single center experience from China. J Gastrointest Surg. 2012; 16: 1123-7. doi:10.1007/s11605-012-1881-y.

3. Wilson DM, 3rd, Bohr VA. The mechanics of base excision repair, and its relationship to aging and disease. DNA Repair (Amst). 2007; 6: 544-59. doi:10.1016/j.dnarep.2006.10.017.

4. Barakat K, Gajewski M, Tuszynski JA. DNA repair inhibitors: the next major step to improve cancer therapy. Curr Top Med Chem. 2012; 12: 1376-90. doi:CTMC-12-12-1376 [pii].

5. Masaoka A, Horton JK, Beard WA, Wilson SH. DNA polymerase beta and PARP activities in base excision repair in living cells. DNA Repair (Amst). 2009; 8: 1290-9. doi:10.1016/j.dnarep.2009.08.004.

6. Zmudzka BZ, Fornace A, Collins J, Wilson SH. Characterization of DNA polymerase beta mRNA: cell-cycle and growth response in cultured human cells. Nucleic Acids Res. 1988; 16: 9587-96.

7. Dalal S, Chikova A, Jaeger J, Sweasy JB. The Leu22Pro tumor-associated variant of DNA polymerase beta is dRP lyase deficient. Nucleic Acids Res. 2008; 36: 411-22. doi:10.1093/nar/gkm1053.

8. Canitrot $Y$, Hoffmann JS, Calsou P, Hayakawa H, Salles B, Cazaux C. Nucleotide excision repair DNA synthesis by excess DNA polymerase beta: a potential source of genetic instability in cancer cells. FASEB J. 2000; 14: 1765-74.

9. Srivastava DK, Husain I, Arteaga CL, Wilson SH. DNA polymerase beta expression differences in selected human tumors and cell lines. Carcinogenesis. 1999; 20: 1049-54.
10. Bhattacharyya N, Chen HC, Comhair S, Erzurum SC, Banerjee S. Variant forms of DNA polymerase beta in primary lung carcinomas. DNA Cell Biol. 1999; 18: 549-54. doi:10.1089/104454999315097.

11. Dobashi Y, Shuin T, Tsuruga H, Uemura H, Torigoe S, Kubota Y. DNA polymerase beta gene mutation in human prostate cancer. Cancer Res. 1994; 54: 2827-9.

12. Miyamoto H, Miyagi $Y$, Ishikawa T, Ichikawa $Y$, Hosaka M, Kubota Y. DNA polymerase beta gene mutation in human breast cancer. Int J Cancer. 1999; 83: 708-9. doi:10.1002/(SICI)1097-0215(19991126)83:5<708::AID-IJC24>3.0.CO;2-C [pii].

13. Wang L, Patel U, Ghosh L, Banerjee S. DNA polymerase beta mutations in human colorectal cancer. Cancer Res. 1992; 52: 4824-7.

14. Zhao GQ, Wang T, Zhao Q, Yang HY, Tan XH, Dong ZM. Mutation of DNA polymerase beta in esophageal carcinoma of different regions. World J Gastroenterol. 2005; 11: 4618-22.

15. Tan XH, Zhao M, Pan KF, Dong Y, Dong B, Feng GJ, et al. Frequent mutation related with overexpression of DNA polymerase beta in primary tumors and precancerous lesions of human stomach. Cancer Lett. 2005; 220: 101-14. doi:10.1016/j.canlet.2004.07.049.

16. Betsalel OT, van de Kamp JM, Martinez-Munoz C, Rosenberg EH, de Brouwer AP, Pouwels PJ, et al. Detection of low-level somatic and germline mosaicism by denaturing high-performance liquid chromatography in a EURO-MRX family with SLC6A8 deficiency. Neurogenetics. 2008; 9: 183-90. doi:10.1007/s10048-008-0125-5.

17. Ponti G, Losi L, Martorana D, Priola M, Boni E, Pollio A, et al. Clinico-pathological and biomolecular findings in Italian patients with multiple cutaneous neurofibromas. Hered Cancer Clin Pract. 2011; 9: 6. doi:10.1186/1897-4287-9-6.

18. Tang Z, Zhao M, Ji J, Yang G, Hu F, He J, et al. Overexpression of gastrin and c-met protein involved in human gastric carcinomas and intestinal metaplasia. Oncol Rep. 2004; 11: 333-9.

19. Tan X, Anzick SL, Khan SG, Ueda T, Stone G, Digiovanna JJ, et al. Chimeric negative regulation of $\mathrm{p} 14 \mathrm{ARF}$ and TBX1 by a $\mathrm{t}(9 ; 22)$ translocation associated with melanoma, deafness, and DNA repair deficiency. Hum Mutat. 2013; 34: 1250-9. doi:10.1002/humu.22354.

20. Tan X, Peng J, Fu Y, An S, Rezaei K, Tabbara S, et al. miR-638 mediated regulation of BRCA1 affects DNA repair and sensitivity to UV and cisplatin in triple negative breast cancer. Breast cancer research : BCR. 2014; 16: 435. doi:10.1186/s13058-014-0435-5.

21. Fu Y, Lian Y, Kim KS, Zhang L, Hindle AK, Brody F, et al. BP1 Homeoprotein Enhances Metastatic Potential in ER-negative Breast Cancer. Journal of Cancer. 2010; 1: 54-62.

22. Marti-Renom MA, Stuart AC, Fiser A, Sanchez R, Melo F, Sali A. Comparative protein structure modeling of genes and genomes. Annu Rev Biophys Biomol Struct. 2000; 29: 291-325. doi:10.1146/annurev.biophys.29.1.291.

23. Batra VK, Perera L, Lin P, Shock DD, Beard WA, Pedersen LC, et al. Amino acid substitution in the active site of DNA polymerase beta explains the energy barrier of the nucleotidyl transfer reaction. J Am Chem Soc. 2013; 135: 8078-88. doi:10.1021/ja403842j.

24. Kleywegt GJ, Jones TA. Model building and refinement practice. Methods Enzymol. 1997; 277: 208-30.

25. Kleywegt GJ, Jones TA. Model-building and refinement practice. Methods In Enzymology. 1999; 277: 173.

26. Barakat K, Tuszynski J. Relaxed complex scheme suggests novel inhibitors for the lyase activity of DNA polymerase beta. J Mol Graph Model. 2011; 29: 702-16. doi:10.1016/j.jmgm.2010.12.003.

27. Poltoratsky V, Prasad R, Horton JK, Wilson SH. Down-regulation of DNA polymerase beta accompanies somatic hypermutation in human BL2 cell lines. DNA Repair (Amst). 2007; 6: 244-53. doi:10.1016/j.dnarep.2006.10.003.

28. Singhal RK, Prasad R, Wilson SH. DNA polymerase beta conducts the gap-filling step in uracil-initiated base excision repair in a bovine testis nuclear extract. J Biol Chem. 1995; 270: 949-57.

29. Starcevic D, Dalal S, Sweasy JB. Is there a link between DNA polymerase beta and cancer? Cell Cycle. 2004; 3: 998-1001. doi:1062 [pii].

30. Sweasy JB, Lang T, DiMaio D. Is base excision repair a tumor suppressor mechanism? Cell Cycle. 2006; 5: 250-9. doi:2414 [pii].

31. Kasakura Y, Mochizuki F, Wakabayashi K, Kochi M, Fujii M, Takayama T. An evaluation of the effectiveness of extended lymph node dissection in patients with gastric cancer: a retrospective study of 1403 cases at a single institution. J Surg Res. 2002; 103: 252-9. doi:10.1006/jsre.2002.6368.

32. Usmani BA, Lunec J, Sherbet GV. DNA repair and repair fidelity in metastatic variants of the B16 murine melanoma. J Cell Biochem. 1993; 51: 336-44. doi:10.1002/jcb.240510313.

33. Wei Q, Cheng L, Xie K, Bucana CD, Dong Z. Direct correlation between DNA repair capacity and metastatic potential of K-1735 murine melanoma cells. J Invest Dermatol. 1997; 108: 3-6. doi:S0022202X97819276 [pii]

34. Shadan FF, Villarreal LP. Potential role of DNA polymerase beta in gene therapy against cancer: a case for colorectal cancer. Med Hypotheses. 1996; 47: 1-9. doi:S0306-9877(96)90033-X [pii].

35. Sugo N, Niimi N, Aratani Y, Takiguchi-Hayashi K, Koyama H. p53 Deficiency rescues neuronal apoptosis but not differentiation in DNA polymerase beta-deficient mice. Mol Cell Biol. 2004; 24: 9470-7. doi:10.1128/MCB.24.21.9470-9477.2004. 
36. Hoffmann JS, Pillaire MJ, Maga G, Podust V, Hubscher U, Villani G. DNA polymerase beta bypasses in vitro a single $\mathrm{d}(\mathrm{GpG})$-cisplatin adduct placed on codon 13 of the HRAS gene. Proc Natl Acad Sci U S A. 1995; 92: 5356-60.

37. Horton JK, Watson M, Stefanick DF, Shaughnessy DT, Taylor JA, Wilson SH. XRCC1 and DNA polymerase beta in cellular protection against cytotoxic DNA single-strand breaks. Cell Res. 2008; 18: 48-63. doi:10.1038/cr.2008.7.

38. Kraulis PJ. MOLSCRIPT: a program to produce both detailed and schematic plots of protein structures. Journal of Applied Crystallography. 1991; $24: 5$. 